\title{
The ENUBET narrow band neutrino beam
}

\author{
F. Pupilli* \\ INFN Sezione di Padova, via Marzolo 8 - Padova, Italy \\ E-mail: fabio.pupilliepd.infn.it
}

F. Acerbi ${ }^{o}$, G. Ballerini ${ }^{a, b}$, M. Bonesini ${ }^{b}$, C. Brizzolari ${ }^{a}, b$, G. Brunetti ${ }^{j}$, M. Calviani $^{m}$, S. Carturan ${ }^{t}$, M. G. Catanesi ${ }^{l}$, S. Cecchini ${ }^{c}$, F. Cindolo ${ }^{c}$, G. Collazuol ${ }^{k, j}$, E. Conti ${ }^{j}$, F. Dal Corso ${ }^{j}$, G. De Rosa ${ }^{p, q}$, C. Delogu ${ }^{b, h}$, A. Falcone ${ }^{j, k}$, B. Goddard ${ }^{m}$, A. Gola $^{o}$, R. A. Intonti ${ }^{l}$, C. Jollet ${ }^{d}$, V. Kain ${ }^{m}$, B. Klicek ${ }^{u}$, Y. Kudenko ${ }^{r}$, M. Laveder ${ }^{k, j}$, A. Longhin ${ }^{j}$, P.F. Loverre ${ }^{n, f}$, L. Ludovici ${ }^{f}$, L. Magaletti ${ }^{l}$, G. Mandrioli ${ }^{c}$, A. Margotti $^{c}$, V. Mascagna ${ }^{a, b}$, N. Mauri ${ }^{c}$, A. Meregagliaa ${ }^{s}$ M. Mezzetto ${ }^{j}$, M. Nessi ${ }^{m}$, A. Paoloni ${ }^{e}$, M. Pari ${ }^{k, j}$, E. Parozzi ${ }^{b, h}$, L. Pasqualini ${ }^{c, g}$, G. Paternoster ${ }^{o}$, L. Patrizii ${ }^{c}$, C. Piemonte ${ }^{o}$, M. Pozzato ${ }^{c}$, M. Prest ${ }^{a, b}$, E. Radicioni ${ }^{l}$, C. Riccio ${ }^{p, q}$, A. C. Ruggieri ${ }^{p}$, G. Sirri ${ }^{c}$, M. Soldani ${ }^{a, b}$, M. Stipcevic ${ }^{u}$, M. Tenti ${ }^{b, h}$, F. Terranova ${ }^{a, b}$, M. Torti ${ }^{b, h}$, E. Vallazza ${ }^{i}$, F. Velotti ${ }^{m}$, M. Vesco $^{t}$, L. Votano ${ }^{e}$

${ }^{a}$ Phys. Dep., Università degli studi dell'Insubria, via Valeggio 11, Como, Italy

${ }^{b}$ INFN, Sezione di Milano-Bicocca, piazza della Scienza 3, Milano, Italy,

${ }^{c}$ INFN, Sezione di Bologna, viale Berti-Pichat 6/2, Bologna, Italy

${ }^{d}$ IPHC, Université de Strasbourg, CNRS/IN2P3, Strasbourg, France

e INFN, Laboratori Nazionali di Frascati, via Fermi 40, Frascati (Rome), Italy

${ }^{f}$ INFN, Sezione di Roma 1, piazzale A. Moro 2, Rome, Italy

${ }^{g}$ Phys. Dep. Università di Bologna, viale Berti-Pichat 6/2, Bologna, Italy

${ }^{h}$ Phys. Dep. Università di Milano-Bicocca, piazza della Scienza 3, Milano, Italy

${ }^{i}$ INFN Sezione di Trieste, via Valerio, 2 - Trieste, Italy

j INFN Sezione di Padova, via Marzolo, 8 - Padova, Italy

${ }^{k}$ Phys. Dep. Università di Padova, via Marzolo, 8 - Padova, Italy

${ }^{l}$ INFN Sezione di Bari, via Amendola, 173 - Bari, Italy

${ }^{m}$ CERN, Geneva, Switzerland

${ }^{n}$ Phys. Dep. Università La Sapienza, piazzale A. Moro 2, Rome, Italy

${ }^{o}$ Fondazione Bruno Kessler (FBK) and INFN TIFPA, Trento, Italy

${ }^{p}$ INFN, Sezione di Napoli, via Cinthia, 80126, Napoli, Italy

${ }^{q}$ Phys. Dep. Università degli Studi di Napoli Federico II, via Cinthia, 80126, Napoli, Italy

${ }^{r}$ Institute of Nuclear Research of the Russian Academy of Science, Moscow, Russia

${ }^{s}$ CENBG, Université de Bordeaux, CNRS/IN2P3, 33175 Gradignan, France

${ }^{t}$ INFN Laboratori Nazionali di Legnaro, Viale dell'Università, 2 - Legnaro (PD), Italy

${ }^{u}$ Center of Excellence for Advanced Materials and Sensing Devices, Ruder Boskovic Institute, Zagreb, Croatia 
ENUBET has been conceived to monitor positrons from $K_{e 3}$ decays in conventional neutrino beam decay tunnels in order to provide a direct determination of the $v_{e}$ flux at the source, paving the way to a measurement of electron neutrino cross section with $1 \%$ precision. Since 2016 remarkable results on the prototyping and testing of the tunnel instrumentation for positron tagging have been achieved, as well as on the design and simulation of the proton extraction scheme, the focusing system and the meson transfer line. In particular the demonstration of the viability of a static focusing extend the potential of monitored neutrino beams beyond the original aim of ENUBET: the flux measurement at the $1 \%$ level also for $v_{\mu}$ produced in the beam. Particular emphasys will be given on this topic and on the possibility to determine the neutrino energy without relying on final state particle reconstruction, thanks to the narrow momentum width of the selected mesons.

Neutrino Oscillation Workshop (NOW2018)

9 - 16 September, 2018

Rosa Marina (Ostuni, Brindisi, Italy)

${ }^{*}$ Speaker. 


\section{Monitored neutrino beams and the ENUBET approach}

The main limitation for a precise neutrino cross section measurement is nowadays represented by the knowledge of the initial flux, since it is not measured in a direct way but it relies on hadroproduction data and on a detailed simulation of the neutrino beamline. The direct monitoring of the neutrino associated charged lepton in the decay tunnel can overcome this limitation [1]. The main goal of the ERC project ENUBET ("Enhanced NeUtrino BEams from kaon Tagging") [2, $3]$ is to study all the accelerators challenges of a facility in which the only source of electron (anti-)neutrinos is the three body semileptonic kaon decay $\left(K^{+(-)} \rightarrow \pi^{0} e^{+(-)} v_{e}\left(\bar{v}_{e}\right)\right)$ and to build detectors to identify the associated positrons in the harsh environment of a neutrino decay tunnel. In the ENUBET narrow band beam concept the meson transfer line $(\sim 20 \mathrm{~m})$ and the decay tube $(40 \mathrm{~m})$ are short and the momentum of the selected mesons $(8.5 \mathrm{GeV} / \mathrm{c} \pm 10 \%)$ is large, in order to minimize the $v_{e}$ contamination from muon decays in flight and the fraction of kaons decaying before the tunnel instrumentation. Unlike most of the beams currently in operation, the decay tunnel is not placed in front of the focusing system and the proton extraction length is slow (a few $\mathrm{ms}$ in the horn option and a few $\mathrm{s}$ in the static focusing option). This ensures that the rate of offmomentum particles, mostly due to low energy products of tertiary interactions in the collimators, is many orders of magnitude smaller than those of currently operating beams and the production of particles (e.g. positrons from kaons) in the decay tunnel can be monitored at single particle level by instrumenting a fraction of the decay tunnel.

\section{Beamline and tunnel instrumentation}

Single-particle monitoring is possible only if the duration of the proton extraction is slower than current long-baseline neutrino beams (tens of $\mu \mathrm{s}$ ) in order to keep the particle rate in the decay tunnel well below $1 \mathrm{MHz} / \mathrm{cm}^{2}$. The maximum spill duration depends on the implementation of the focusing system for the particles produced in the target. Horn magnets cannot be pulsed longer than $\sim 10 \mathrm{~ms}$, while a quadrupole based system can be operated in DC mode so that the proton extraction can last several seconds. ENUBET is studying both options and developing a "burst-mode" extraction at the ms scale and a "slow" extraction mode with a duration of $\mathscr{O}(1 \mathrm{~s})$. In the burst-mode many consecutive spills (few ms long) are extracted within one macro SPS spill ( $\sim 4 \mathrm{~s})$. This scheme, allowing the use of a horn focusing system in ENUBET, has been already implemented and tested in the SPS as a proof-of-concept before the LHC Long Shutdown 2 and it will be further tuned and validated at the restart of the CERN accelerator complex in 2021.

Primary proton interactions on target were simulated with FLUKA 2011, considering the energies of the main existing proton drivers $(30,120,400 \mathrm{GeV})$. The transfer line optics has been optimized using TRANSPORT for a central momentum of $8.5 \mathrm{GeV}$ and a momentum bite of $10 \%$, in both the horn and static focusing options. The latter turned out to perform significantly better (a factor 4 increase in the meson yield at the tunnel) than early estimates reported in the ENUBET proposal [1]. It consists of a quadrupole triplet placed directly downstream of the ENUBET target, followed by a bending dipole magnet of $1.8 \mathrm{~T}$ providing a bending angle of $7.4^{\circ}$, and by another quadrupole triplet before the decay tunnel. This configuration has been implemented in G4Beamline, including also absorber and collimator elements. Assuming the SPS as driver (400 
$\mathrm{GeV}$ protons), the pion and kaon yields in the reference momentum bite amount to $19 \times 10^{-3}$ and $1.4 \times 10^{-3}$ particles per pot (proton on target) respectively, a factor $4 \div 6$ smaller than in the hornbased configuration [4]. However, the longer extraction made possible by the static focusing allows for a reduction of the particle rate in the decay tunnel, thus reducing the pile-up for charged decay products identification. Furthermore, it could ease the implementation of a time tagged beam where the $v_{e}$ at the detector is time-correlated with the produced $\mathrm{e}^{+}$in the decay tunnel [5].

The ENUBET instrumented decay tunnel consists of a calorimeter for $\mathrm{e}^{+} / \pi^{+}$separation and of an inner light-weight detector for $\mathrm{e}^{+} / \pi^{0}$ separation and timing (" $t_{0}$-layer"). During 2016-2018 test beam exposures in four two-week slots of data taking were performed to validate prototypes for the $t_{0}$-layer and the calorimeters both in shashlik and lateral readout modes $[3,6,7,8]$. At present, both options fulfill the requirements of ENUBET and a final decision will be taken in 2019 based on the result of the tests done at CERN in October 2018, currently under analysis. Particles inside the tunnel are identified by the energy deposition pattern in the calorimeter and photon veto. The positrons are selected with a signal-to-noise ratio of $\sim 0.5$ and an efficiency of $\sim 20 \%$, which is appropriate for the neutrino flux monitoring at the per-cent level [5]. This level of reconstruction is achieved for the static focusing system assuming a complete extraction of the protons from the SPS in a super-cycle $\left(4.5 \times 10^{13}\right.$ pot in a 2 s slow extraction).

\section{The ENUBET narrow band beam}

Assuming $4.5 \times 10^{19}$ pot at CERN-SPS and a static focusing configuration, about $1.16 \times 10^{6}$ $v_{\mu}^{C C}$ and $1.4 \times 10^{4} v_{e}^{C C}$ interactions will be collected at a neutrino detector of 500 ton mass and $6 \times 6 \mathrm{~m}^{2}$ transverse size located $50 \mathrm{~m}$ from the end of the decay tunnel.

The monitoring of $\mathrm{e}^{+}$in the decay tunnel provides the measurement of the $v_{e}$ flux, while, in the static focusing configuration with a slow extraction $(2 \mathrm{~s})$, a precise determination of the $v_{\mu}$ flux too can be achieved by measuring the $\mu$ rate after the hadron dump at single particle level.

Furthermore ENUBET can provide a measurement of the neutrino energy that does not rely on the reconstruction of final state particles. This feature ("narrow-band off-axis technique") results from the narrow momentum bandwidth of the beam and the finite transverse dimension of the neutrino detector, and it can be exploited by neutrino detectors that have a size comparable or larger than ICARUS at Fermilab or the ProtoDUNE detectors at CERN.

The energy measurement is based on the correlation between the energy of the neutrino interacting in the detector and the radial distance $(\mathrm{R})$ of the interaction vertex from the beam axis. By selecting interactions in radial windows of $\pm 10 \mathrm{~cm}$ at $\mathrm{R}$ equal to $0.5 \mathrm{~m}, 1.5 \mathrm{~m}$ and $2.5 \mathrm{~m}$ (Fig. 1, right plot), we collect samples of about $15.7 \times 10^{4}, 6.4 \times 10^{4}$ and $2.9 \times 10^{4} v_{\mu}^{C C}$ events respectively in the energy range of interest for future long baseline oscillation experiments.

The incoming neutrino energy can be determined with a precision given by the pion peak width of the spectrum at a fixed $\mathrm{R}$. It ranges from $7 \%$ at $3.5 \mathrm{GeV}$ to $22 \%$ at $0.8 \mathrm{GeV}$ as shown in Fig. 2.

\section{References}

[1] A. Longhin, L. Ludovici and F. Terranova, Eur. Phys. J. C 75, 155 (2015). 

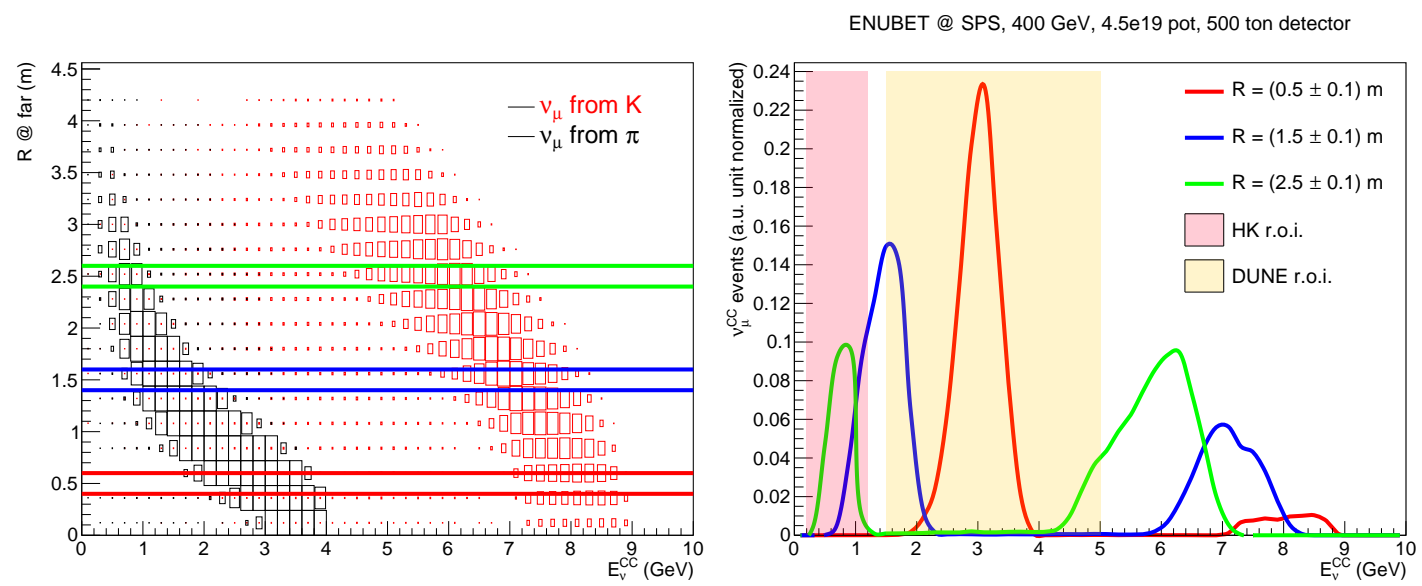

Figure 1: (Left) Radial and energy distribution of $v_{\mu}^{C C}$ interactions. The high energy neutrino component from kaon decays is shown with red boxes, while the low energy one from pions corresponds to the black boxes. (Right) $v_{\mu}^{C C}$ spectra normalized to unity obtained selecting interactions at different radial distances from the beam axis.

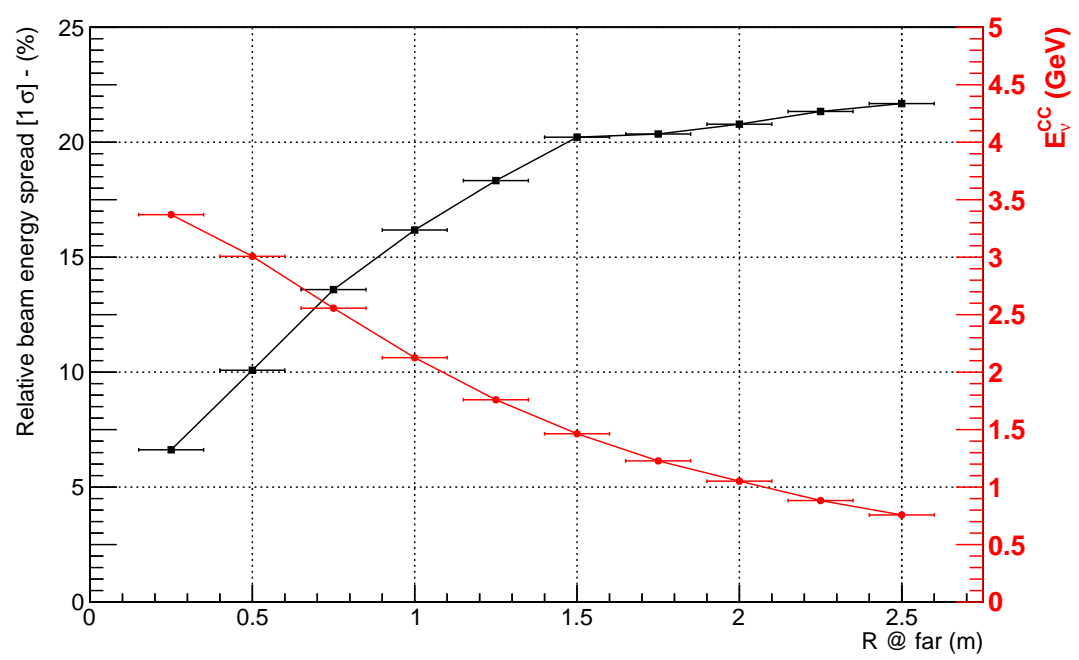

Figure 2: Beam energy spread (in black) and peak energy (in red) as a function of the distance R of the interaction vertex at the detector from the beam axis.

[2] A. Berra et al., CERN-SPSC-2016-036; SPSC-EOI-014.

[3] Documentation available at http://enubet.pd.infn.it

[4] G. Brunetti, Talk at 20th International Workshop on Neutrinos from Accelerators (NUFACT2018), Blacksburg, Virginia, August 12-18, 2018.

[5] F. Acerbi et al., CERN-SPSC-2018-034; SPSC-I-248.

[6] A. Berra et al., Nucl. Instrum. Meth. A 830 (2016) 345.

[7] A. Berra et al., IEEE Trans. Nucl. Sci. 64 (2017) 1056.

[8] G. Ballerini et al., JINST 13 (2018) P01028. 